\title{
OPTIMIZACIÓN DE LA TASA DE PENETRACIÓN MEDIANTE EL ANÁLISIS DE LAS VIBRACIONES AL PERFORAR, CASO DE ESTUDIO ECUADOR
}

\author{
Diego Ayala*; Alina Benítez; Raúl Valencia \\ Facultad de Ingeniería en Geología y Petróleos. Escuela Politécnica Nacional. PO.Box 17-01-2759. Quito. Ecuador. \\ *E.mail: diego.ayala.t@gmail.com
}

\section{RESUMEN}

La exposición a las vibraciones en la construcción de un pozo es un fenómeno inherente a la perforación, las geometrías cada vez más complejas de los pozos, así como la dureza de las formaciones atravesadas son algunos de los factores que propician condiciones desfavorables y potencian un fallo prematuro de las herramientas de perforación. El estudio expone el proceso de optimización llevado a cabo para perforar la formación Napo (Cuenca Oriente) en la sección de 12 1/4”, mediante el uso de sensores de vibración para registrar la dinámica de perforación; el análisis se realizó en tres pozos, todos ellos con geometría tipo "J". La correcta interpretación de los registros y la implementación de las recomendaciones que dieron como resultado del análisis pozo a pozo, permitieron realizar cambios en el diseño del BHA y establecer parámetros de perforación, que resultan en menores niveles de vibración y por lo tanto en mejores desempeños.

Palabras clave: Vibraciones al perforar, Optimización de la perforación, Problemas al perforar.

\section{OPTIMIZATION OF RATE OF PENETRATION BY VIBRATION ANALYSIS, CASE STUDY ECUADOR}

\begin{abstract}
Exposure to vibrations in the oil well construction is an inherent phenomenon of drilling operation, the increasingly complex geometries of the wells, the hardness of the formations crossed are factors that cause unfavorable conditions and promote a failure of the drilling tools. The study outlines the optimization process carried out to drill Napo Formation (Cuenca Oriente) in $12 \frac{1}{4}$ " section using vibration sensors to record the drilling dynamics; the analysis was performed in three directional oil wells, the correct interpretation of the records and the implementation of the recommendations that resulted from the well-to-well analysis allowed to make changes in the design of the BHA and to establish parameters of drilling, which result in lower levels of vibration and therefore in better performances .
\end{abstract}

Keywords: Drilling optimization, Drilling problems, Drilling vibrations.

Cita: Ayala, D., Benítez, A. y Valencia, R. (2017). Optimización de la tasa de penetración mediante el análisis de las vibraciones al perforar, caso de estudio Ecuador. Revista Fuentes: El reventón energético, 15 (1), 27-40. 


\section{INTRODUCCIÓN}

Una broca que no se adecua correctamente a la formación, los parámetros de perforación, el BHA, o las herramientas de fondo de pozo, puede introducir aspectos dinámicos indeseados $\mathrm{o}$ generar fuerzas que produzcan la desviación del pozo respecto de la trayectoria planificada. Por el contrario, una barrena diseñada correctamente genera un pozo más calibrado $\mathrm{y}$ un trayecto menos tortuoso. (Centale, y otros, 2011)

Los especialistas pueden diseñar una broca que se acople de mejor manera a la formación y evitar de ese modo las ROP bajas o el tiempo no productivo excesivo causado por los viajes requeridos para reemplazar las brocas gastadas. (Ayala, Torres, Valencia y Loaiza, 2016).

La perforación de la sección de 12 1/4"que corresponde a la Formación NAPO (sucesión de lutitas negras, calizas grises a negras y areniscas calcáreas, espesor de 200 a 700 metros (Baldock, 1982), es el intervalo más crítico durante la perforación, esta sección se la puede dividir en dos intervalos, un primer intervalo, donde se perfora las formaciones Tiyuyacu y Tena (arcillolita y entre dos y tres capas de conglomerado, en el caso de Tiyuyacu (Bady, Rivadeneira, y Barragan, 2004), este intervalo ha sido plenamente optimizado y no representa ningún problema. En el segundo intervalo se perfora la formación Napo, al tratarse de una formación intercalada es muy propensa a generar vibraciones y varios problemas han sido identificados en este intervalo los cuales son:

- Daño prematuro de las brocas de perforación. Dos brocas necesarias para perforar Napo.

- Alta vibración torsional causando dificultades para direccionar el pozo.

- Daño de las herramientas de fondo.

- Baja ROP debido al desgaste de la estructura de corte de la broca.

- Dificultades durante los viajes a causa de la tortuosidad ocasionada por los problemas direccionales.

Varios ensamblajes de fondo y tipos de drive se probaron para perforar la formación Napo, obteniéndose resultados nada satisfactorios.

- Con Motor, los problemas de transferencia de peso y estoleamiento se traducían en ROP muy bajas (menores a $37 \mathrm{ft} / \mathrm{hr}$ ), se observó un daño considerable de la estructura de corte de la broca, con evidencia de impacto y daño térmico de los cortadores.

- Utilizando RSS, la vibración torsional dificultaban considerablemente mantener la dirección del pozo por los altos niveles de vibración, la broca presenta un desgaste importante debido a la degradación térmica y daño mecánico, razón por la cual de manera reiterada se interrumpió la perforación para realizar cambios en el BHA y broca.

El objetivo de optimizar radica en mejorar el diseño de las brocas, para reducir el desgaste y el número de brocas utilizadas, la optimización proporcionará un ahorro en tiempos de viajes y de perforación al incrementar la ROP. Los antecendetes de otros pozos, evidencian fuertes niveles vibracionales, por ésta razón, es importante entenderlas, encontrar sus causas y mitigarlas para conseguir una perforación eficiente.

\subsection{PROBLEMAS ASOCIADOS A LAS VIBRACIONES}

\subsubsection{REBOTE DE BROCA}

La acción de corte de las brocas de 3 conos tiende a crear formas lobulares en el fondo del pozo en formaciones duras, lo que hace que la broca sea desplazada axialmente 3, 6 o incluso 9 veces por cada revolución, modificando el peso efectivo sobre la barrena.

Durante este fenómeno, la broca raramente rebota del fondo del pozo, más bien, se lo debe entender como una fluctuación en el valor de peso aplicado sobre la broca de forma periódica (DATALOG, 2001).

\subsection{2. "STICK-SLIP"}

El atascamiento deslizamiento, es un caso severo de vibración torsional, donde la broca permanece estacionaria por un periodo de tiempo debido a la fricción producida entre esta y la formación. Cuando el esfuerzo de torsión dentro de la sarta de perforación es mayor que estas fuerzas de fricción, la broca se suelta de la pared del pozo y gira por la acción de destornillado de la columna de perforación genera un movimiento lateral destructivo.

Según los operadoras de perforación, el fenómeno de atascamiento deslizamiento da cuenta de aproximadamente un 50\% del tiempo de perforación en el fondo del pozo. (Xianping, Páez, Partin y Agrinhorti, 2010). 
Las soluciones más frecuentes usadas en el campo para la reducción del fenómeno de atascamiento deslizamiento son: el aumento de la velocidad de rotación, el descenso del WOB o la modificación de las características de los lodos de perforación. (Sananikone, Kamoshima y White, 1992)

\subsubsection{REMOLINO}

El efecto remolino "whirl", ocurre cuando hay poco peso sobre la broca o el número de RPM es muy alto para el tipo de formación geológica. Cuando se perfora un hoyo en calibre, la broca gira alrededor de su propio centro; pero durante un movimiento en forma de remolino el centro instantáneo de rotación pasa a ser un cortador en la cara o el calibre de la broca, donde uno de los lados de la broca es empujado contra la pared del pozo por una fuerza centrífuga, incrementándose la fuerza de fricción y con esto el efecto remolino. (Korin, et.al., 2015)

Una de las consecuencias del remolino es la generación de un pozo con una zona de mayor diámetro. Los cortadores se desplazan ocacionando que la broca recorra distancias más largas por revolución que en un pozo calibrado. Estas acciones generan cargas de alto impacto sobre la broca y el BHA. (Brelt, Warren y Behr, 1990).

\subsection{REGISTRO DE LA DINÁMICA DE FONDO}

El presente estudio, tendrá como eje principal la medición de vibraciones torsionales, por ser las más recurrentes durante la perforación con brocas PDC. El uso de brocas roller cone, será exclusivo para la zona superficial de los pozos en análisis, por esta razón la cuantificación de las vibraciones axiales no se llevará a cabo.

En el análisis se empleó tecnología de la compañía NOV Downhole denominada Black Box, basada en sensores de vibración modo memoria, que se pueden ubicar estratégicamente en diferentes componentes del ensamblaje de fondo (BHA) para medir la aceleración lateral y torsional (National Oilwell Varco, 2007).

El registro obtenido con estos sensores, es empatado con la información de geología, para realizar un análisis completo de la relación entre la dinámica de fondo y las distintas variables involucradas en la perforación. Fueron empleados 2 tipos de sensores: Tipo 1 y Tipo 2 .

\subsubsection{TIPO 1}

Dispositivo de memoria, mide la aceleración de la componente donde fue situado. Con la señal de aceleración centrípeta se obtiene el valor denominado Delta RPM, que es el porcentaje de desviación de las RPM de la señal con respecto a un valor promedio calculado. Con esto se cuantificará la severidad de la vibración torsional de la que es sujeto un determinado punto del BHA. (National Oilwell Varco, 2007)

\subsubsection{TIPO 2}

Dispositivo de memoria que registra la aceleración tangencial y posición angular de un determinado punto para obtener mediante cálculos estadísticos los valores de RMS, impactos laterales, Delta RPM y RPM de fondo. La ventaja que tiene sobre el sensor tipo 1, es que arroja las RPM que se tienen en fondo con mayor precisión, representando de mejor manera el rango dinámico Delta RPM. (National Oilwell Varco, 2007).

\subsection{ANÁLISIS DE LA DINÁMICA DE FONDO}

\subsubsection{CoCA K-29}

Pozo direccional tipo "J", con una inclinación máxima planeada de $28^{\circ}$. Para perforar la formación Napo se utilizó el BHA descrito en la tabla 1, y la conficuración de los sensores detallado en la tabla 2.

Tabla 1. BHA Coca K-29

\begin{tabular}{lc}
\hline \multicolumn{2}{c}{ Información BHA } \\
\hline BHA & Direccional \\
\hline Sistema direccional & RSS \\
Broca & PDC 5 aletas \\
Cortadores & $19 \mathrm{~mm}$ \\
\hline
\end{tabular}

Tabla 2. Sensores Coca K-29

\begin{tabular}{llc}
\multicolumn{3}{c}{ Configuración de Sensores } \\
\hline \multicolumn{1}{c}{ Sensor } & \multicolumn{1}{c}{ Ubicación } & \# Sensores \\
\hline Tipo 2 & Interior broca & 1 \\
Tipo 1 & Sobre monel & 2 \\
Tipo2 & Entre HWDP a 558 ft de la broca & 2 \\
\hline
\end{tabular}

El BHA ingresó a $8412 \mathrm{ft}$ y avanzó hasta $9140 \mathrm{ft}$, después de perforar $728 \mathrm{ft}$ el BHA fue sacado a superficie por tener una baja ROP. La valoración del estado de la broca se indica en la tabla 3. 
Tabla 3. Estado de la Broca

\begin{tabular}{c}
\hline Diagnóstico de la Broca \\
\hline Desgaste considerable por impacto y degradación térmica \\
Código IADC del desgaste de la broca \\
$0-4-W T-S-X-1-C T / H C-P R$ \\
\hline
\end{tabular}

El registro de vibraciones de la figura 1, muestra que se perforó bajo vibración torsional severa en la broca y BHA con acoplamiento de vibración lateral.
- Vibración torsional en la broca (sensor de la broca) superior al $90 \%$, vibración torsional en el BHA (sensor sobre el monel) superior al 50\%.

- Incremento de vibración torsional al perforar caliza $\mathrm{y}$ arenisca.

- Vibración lateral en la broca alrededor de 0.8 (g), y la vibración lateral en el BHA alrededor de $1.8(\mathrm{~g})$.

- Acoplamiento de la vibración lateral con la vibración torsional.

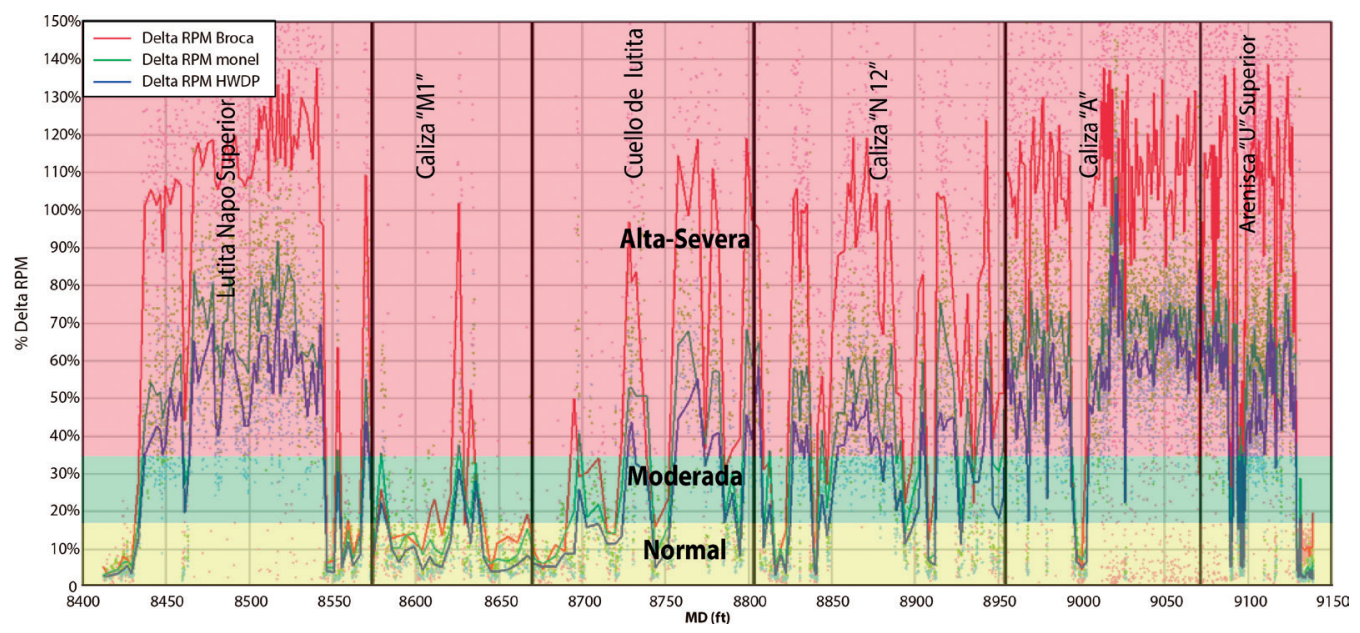

Figura 1. Vibración torsional en MD, Coca K 29.

La intensidad de la vibración torsional indica que la propagación va en el sentido de la broca hacia arriba, en el BHA y en el HWDP, y de acuerdo al comportamiento dependiente de la litología, es evidente que la vibración torsional se está originando de la interacción entre la broca y la formación, la figura 2, muestra que hay una relación entre los parámetros y la vibración torsional.

- Incremento de la vibración torsional conforme incrementa el WOB.

- Reducción de la vibración torsional conforme incrementa las RPM.

- La vibración lateral así como la vibración torsional muestran una misma relación con respecto a los parámetros de perforación.

Con la figura 2, se puede establecer los valores óptimos de WOB y RPM para perforar esta formación y son: WOB menor a 4 klb o RPM mayor a 225 RPM.

Con estas medidas se optimizará el proceso y se reducirán los niveles de vibración torsional, especialmente en la broca.
El WOB que normalmente se requiere para vencer la compresibilidad de la formación, es mucho mayor al necesario para tener vibraciones de intensidad baja. La opción de controlar la vibración torsional por WOB queda descartada; la otra alternativa es incrementar las RPM en la broca por arriba de 200 RPM, esto provee mayor energía a la broca, lo que permite vencer la resistencia de la roca evitando que se detenga la rotación.

Por lo amprendido en el pozo Coca K-29, se implementaran los siguientes cambios en el BHA para el nuevo pozo:

- Cambio de RSS por Motor de Fondo con una salida de torque de media a alta (lobe 7:8, 4 etapas).

- Inclusión de la herramienta de oscilación axial.

La inclusión de la herramienta de oscilación axial se lo hace para reducir la fricción de la sarta de perforación con las paredes del hoyo, que genera problemas de arrastre, baja ROP deslizando y problemas de estoleamiento de motor. 


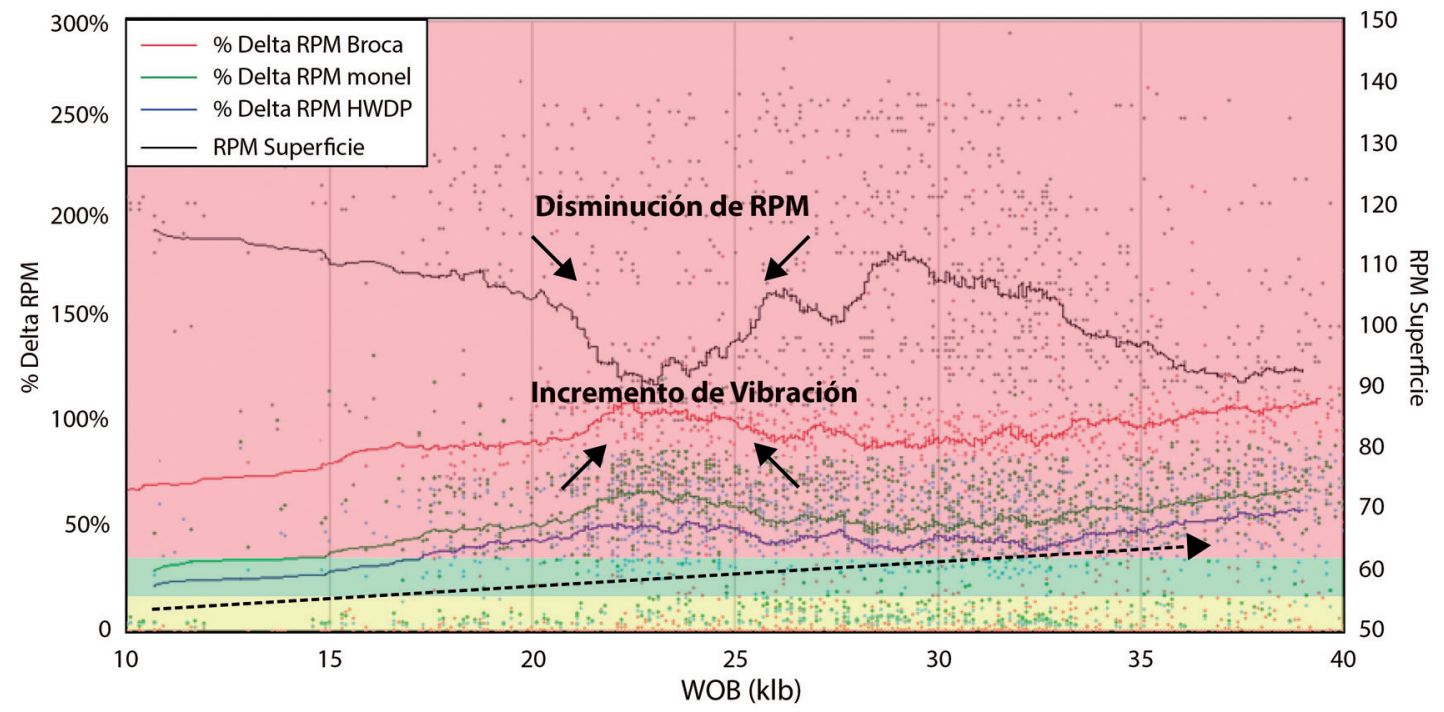

Figura 2. Vibración torsional Vs WOB y RPM de superficie en la Lutita.

\subsubsection{CocA $K-40$}

Pozo direccional tipo "J" con una inclinación máxima planeada de $29.88^{\circ}$. Para perforar la formación Napo se utilizó un BHA fulcro descrito en la tabla 4, y la configuración de los sensores en la tabla 5.

Tabla 4. BHA Coca K-40

\begin{tabular}{lc}
\hline \multicolumn{2}{c}{ Información BHA } \\
\hline BHA & Direccional \\
Sistema direccional & Motor de fondo \\
Broca & PDC 5 aletas \\
Cortadores & $19 \mathrm{~mm}$ \\
\hline
\end{tabular}

Tabla 5. Sensores Coca K-40

\begin{tabular}{llc}
\hline \multicolumn{3}{c}{ Configuración de Sensores } \\
\hline Sensor & \multicolumn{1}{c}{ Ubicación } & \# Sensores \\
\hline Tipo 2 & Interior broca & 1 \\
Tipo 1 & Sobre monel & 2 \\
Tipo2 & $\begin{array}{l}\text { Arriba del estabilizador, } 1257 \mathrm{ft} \\
\text { sobre la broca }\end{array}$ & 2 \\
\hline
\end{tabular}

En este caso, el BHA perforó unos pocos pies de Tena antes de ingresar a Napo, y adicionalmente por necesidad de toma de coring la perforación fue interrumpida, perforando únicamente $681 \mathrm{ft}$, de los cuales $616 \mathrm{ft}$ fueron de Napo.

La figura 3, muestra el registro de vibraciones donde se puede ver que la vibración torsional es mayor en la broca. Mostrando el siguiente comportamiento:
- Vibración torsional en la broca (sensor ubicado en la broca) alrededor de $41 \%$ y en el BHA (sensor ubicado en el monel) alrededor de 19\%.

- Vibración lateral en la broca alrededor de 0.43 (g) y en el BHA alrededor de $0.19(\mathrm{~g})$, no representa ningún problema.

Comparado con el pozo anterior, los niveles de vibración son mucho menores. La vibración torsional en la broca se redujo en $54 \%$, la vibración torsional en el BHA se redujo en $62 \%$. La vibración Lateral en la broca se redujo en $43 \%$ y la vibración lateral en el BHA se redujo en $89 \%$. Esto se tradujo en un incremento de la ROP neta en un $95 \%$.

El desgaste de la broca fue significativamente menor y la valoración del estado de la herramienta se indica en la tabla 6 .

Tabla 6. Estado de la Broca.

\begin{tabular}{c}
\hline Diagnóstico de la Broca \\
Desgaste moderado por impacto y degradación térmica \\
Código IADC del desgaste de la broca \\
0-1-WT-S-X-1-NO-BHA \\
\hline
\end{tabular}

El pozo Coca K-40 estableció los parámetros óptimos para perforar la formación Napo, con un ensamblaje de motor que permite mitigar las vibración torsional y lateral así como obtener mayor ROP. 


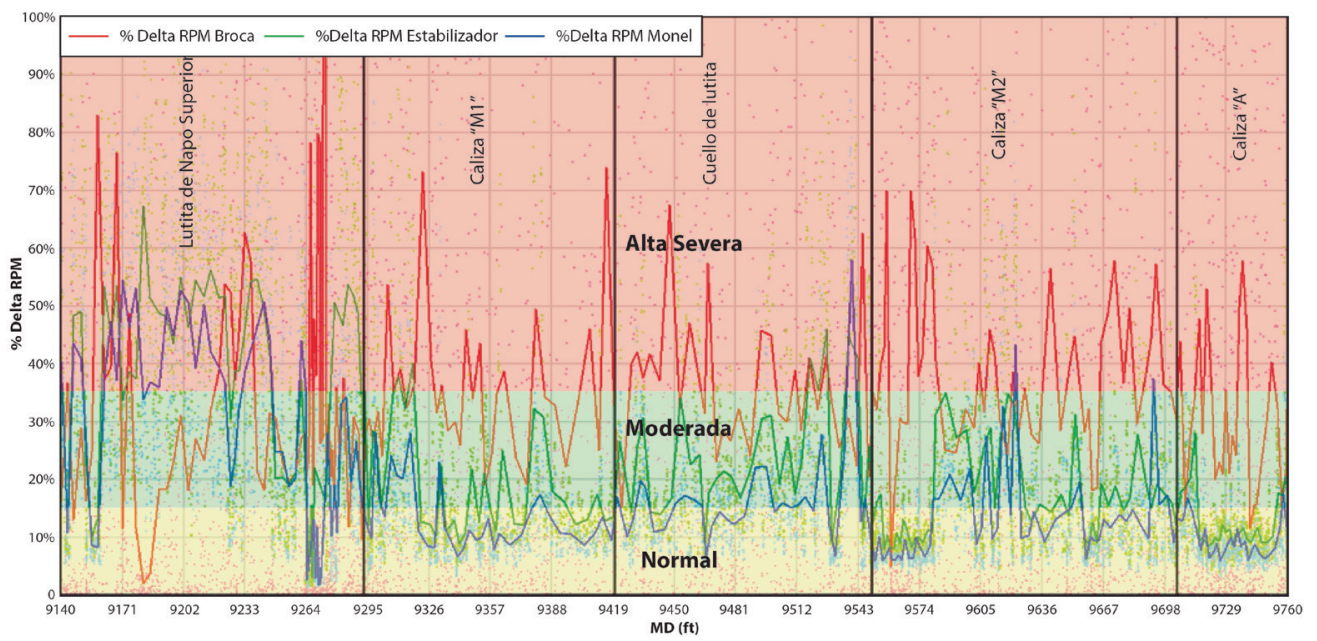

Figura 3. Vibración torsional en MD Coca K-40

\subsubsection{COCA K-41}

Es un pozo direccional tipo "J" de $40.3^{\circ}$ de inclinación máxima. El objetivo direccional en la formación Napo es mantener tangente en $30^{\circ}$. El BHA utilizado es similar al pozo Coca K-40 y se lo detalla en la tabla 7 , y la configuración de los sensores en la tabla 8 .

Tabla 7. BHA Coca K-41

\begin{tabular}{|lc|}
\hline & Información BHA \\
\hline BHA & Direccional \\
\hline Sistema direccional & Motor de fondo \\
\hline Broca & PDC 5 aletas \\
\hline
\end{tabular}

Cortadores

$19 \mathrm{~mm}$
Tabla 8. Sensores Coca K-41.

\begin{tabular}{llc}
\hline \multicolumn{3}{c}{ Configuración de Sensores } \\
\hline Sensor & \multicolumn{1}{c}{ Ubicación } & \# Sensores \\
\hline Tipo 1 & Interior broca & 1 \\
Tipo 1 & Sobre monel & 2 \\
Tipo2 & Arriba de estabilizador, 1245 ft Sobre la broca & 2 \\
\hline
\end{tabular}

El BHA ingresó a $9717 \mathrm{ft}$ y salió a $10964 \mathrm{ft}$. Perforando $1247 \mathrm{ft}$ de la formación Napo e ingresando unos pocos pies en la formación Hollín (arenisca cuarzosa de grano fino,con contenido alto o medio de glauconita (Bady, Rivadeneira y Barragan, 2004). La figura 4, muestra el registro de vibraciones para toda la corrida. Se observa que los niveles de vibración torsional son muy bajos y por lo tanto aceptables.

- Vibración Torsional en la Broca alrededor de 4\% y en el BHA alrededor de $18 \%$.

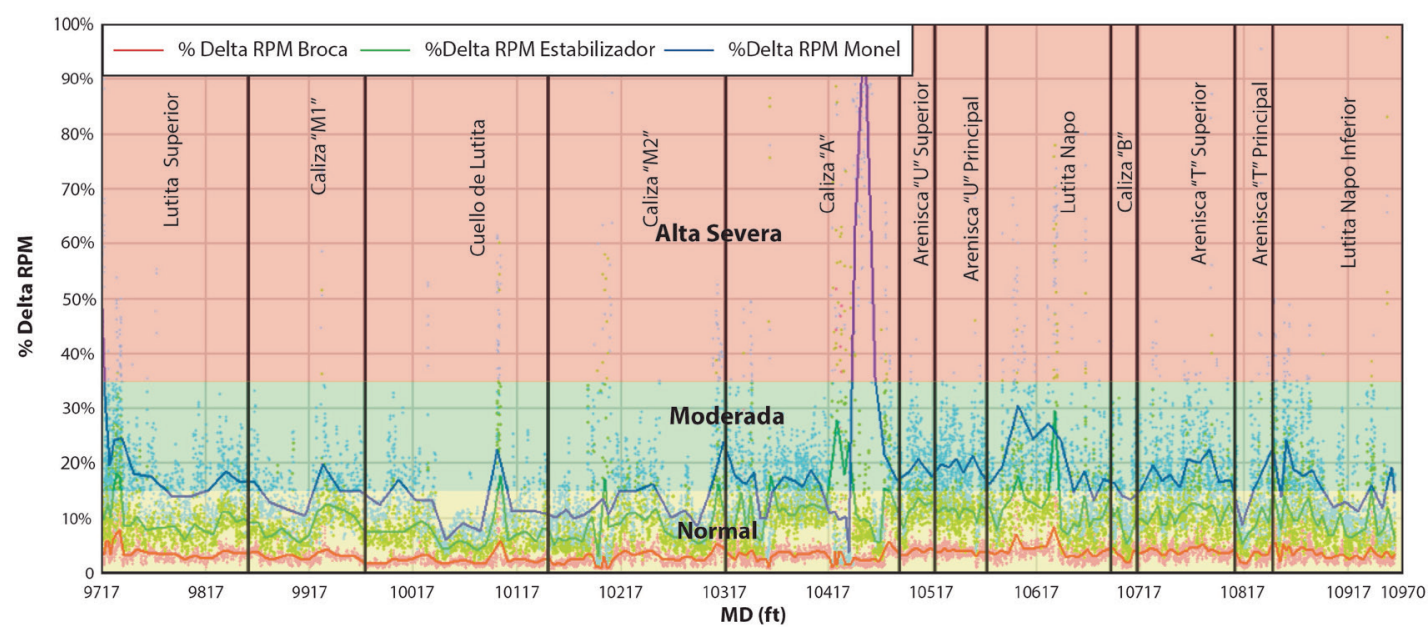

Figura 4. Vibración torsional en MD Coca K-41 
Este resultado se obtuvo al aplicar los parámetros de perforación (RPM y WOB) obtenidos del pozo Coca K-40.

Para esta corrida, se registra un ligero incremento en la vibración lateral, lo cual era de esperarse por los parámetros recomendados, sin embargo los niveles de esta vibración se mantienen en niveles muy bajos.

La vibración torsional en la broca se redujo en $90 \%$ y en el BHA en 5\%. Lo que se refleja en el desgaste de la broca, la valoración del estado de la barrena se indica en la tabla 9 .

Tabla 9. Estado de la Broca.

\section{Diagnóstico de la Broca}

Desgaste moderado por impacto y degradación térmica

Código IADC del desgaste de la broca

0-1-WT-S-X-1-CT-TD

\section{RESULTADOS}

El estudio consideró las recomendaciones de los investigadores de Smith. El análisis de los datos se lo debe realizar en un ambiente de perforación virtual junto con la información relacionada con la broca específica a evaluar, la evaluación debe incluir:

- Localización precisa, propiedades del material y dimensiones de los cortadores.

- Datos dimensionales de los componentes de fondo de pozo y las características físicas de cada elemento del BHA.

- Geometría del pozo propuesto.

- Parámetros operativos planificados (Garret, Teggart y Tetley, 2010).

El análisis incorpora la variación de las vibraciones para la broca y BHA según la profundidad en TVD para los 3 pozos de estudio Coca K-29, Coca K-40 y Coca K-41, donde se puede apreciar la variación de la vibración en los 3 pozos de acuerdo a la litología atravesada.

\subsection{VIBRACIÓN TORSIONAL EN LA BROCA}

La figura 5, recopilados los datos de la vibración torsional que afectó a la broca en profundidad TVD y por sub-formación.

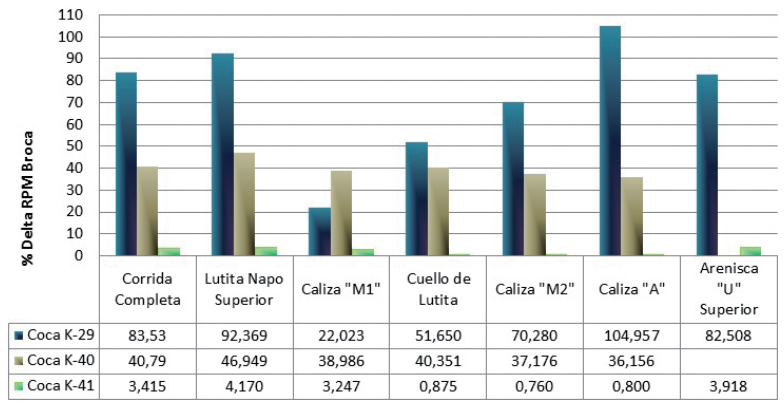

Figura 5. Vibración torsional en la broca según subformaciones.

De la figura 5 se destaca lo siguiente:

- El último pozo perforado Coca K-41, presenta los menores niveles de vibración torsional en la broca y el pozo Coca K-29 es el que registra los niveles más altos de vibración torsional.

- Reducción significante de $51 \%$ de vibración torsional en la broca para el pozo Coca K-40 y del $96 \%$ en el pozo Coca $\mathrm{K}-41$ respecto al pozo inicial Coca K-29.

- Vibración torsional en la broca de nivel normal únicamente en el pozo Coca K-41, lo que permitió un uso eficiente de la energía suministrada a la broca optimizando la perforación.

- Vibración torsional al perforar la caliza "M1", es mayor en el pozo Coca K-40, debido al efecto de resonancia presente en la mayor parte de esta subformación.

- De los paquetes de caliza que conforman Napo, la caliza "A" es la que muestra la mayor intensidad de vibración torsional.

\subsection{VIBRACIÓN TORSIONAL EN EL BHA}

En la figura 6, se presenta la gráfica como resultado de haber tabulado los datos de la vibración torsional que afectó al BHA en profundidad TVD y por subformación: 


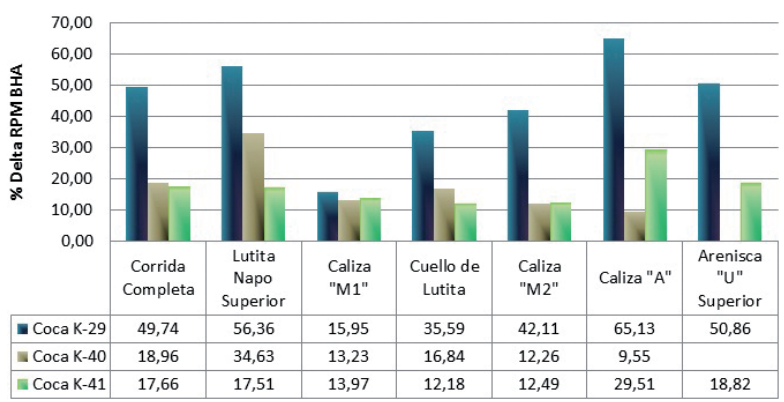

Figura 6. Vibración torsional en el BHA por subformaciones.

Del análisis de los datos de figura 6 se obtuvo:

- Los pozos Coca K-40 y Coca K-41 presentan niveles normales de vibración torsional en el BHA.

- Niveles de vibración torsional en el BHA similares en el pozo Coca K-40 y Coca K-41, siendo el Coca $\mathrm{K}-41$ el que menores niveles registró.

- Reducción del $62 \%$ de la vibración torsional en el pozo Coca K-40 y del 65\% en el Coca K-41.

- Aumento de la actividad torsional en la caliza "A" y la arenisca "U" superior.

- De los paquetes de caliza que conforman Napo, la caliza "A" es la que muestra un mayor nivel de vibraciones torsionales.

\subsection{EVALUACIÓN DE ROP}

La figura 7, muestra el incremento de ROP, de $23 \mathrm{ft} / \mathrm{h}$ en el pozo Coca K-29 a 45 ft/h en el pozo Coca K-40 y llegó a $48 \mathrm{ft} / \mathrm{h}$ en Coca K-41, es decir, un incremento del $109 \%$ de la ROP comparado con el pozo inicial.

El pozo Coca K-41, que muestra el mayor desempeño, estuvo sometido a ciertas condiciones que deben ser consideradas:

- ROP instantánea limitada a $70 \mathrm{ft} / \mathrm{h}$. Esto no permite evaluar cuál es el desempeño que puede alcanzar la broca perforando Napo.

- Mayor inclinación $\left(40.3^{\circ}\right)$ y mayor desplazamiento horizontal que los pozos anteriores.

- Mayor intervalo perforado de Napo, llegando a atravesar toda esta formación, son $500 \mathrm{ft}$ más que se perforaron respecto a los pozos que le anteceden.
- Mayor intervalo deslizado: $43 \mathrm{ft}$ en Coca K-41 y 17 $\mathrm{ft}$ en Coca K-40.

Se logró un incremento de la ROP, muestra de que la dinámica del BHA fue optimizada exitosamente.

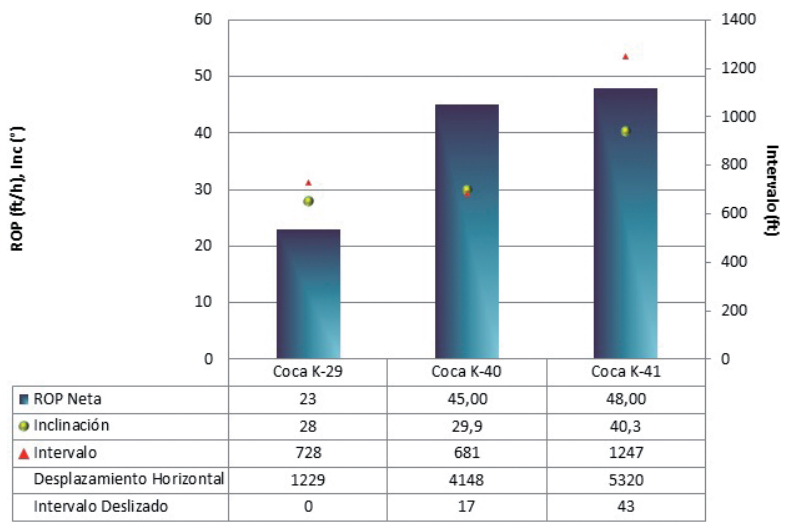

Figura 7. Comparación desempeños Coca K-29, Coca K-40 y Coca K-41.

\subsection{CONDICIÓN DE DESGASTE}

Se analiza la broca para determinar el desgaste y la rotura de la estructura de corte. La industria establece directrices generales para relacionar los patrones de desgaste habituales de las brocas con las posibles causas (Betson y Smith, 1956).

El desgaste de las brocas corridas para perforar Napo en los pozos de estudio, según el código IADC (García, 2008) son los siguientes:

- Coca K-29 con 0-4-WT-S-X-1-CT/HC-PR

- Coca K-40 con 0-1-WT-S-X-I-NO-BHA.

- Coca K-41 con 0-1-WT-S-X-1-CT-TD.

El desgaste mínimo y la buena condición de la broca contribuyeron para lograr una ROP más alta y alcanzar una mejor dinámica de fondo, lo que permitió hacer uso de una sola broca para atravesar toda la formación Napo, eliminando viajes no planificados por cambio de broca.

\subsection{LINEAMIENTOS PARA LA CONFIGURACIÓN DEL BHA}

El proceso iterativo para el desarrollo de configuraciones óptimas de brocas y BHA también es obstaculizado por diversos factores inherentes al proceso. Los ingenieros con distintas experiencias extraen conclusiones disímiles a partir de patrones 
de desgaste esencialmente iguales; por ejemplo, algunos ingenieros pueden identificar la causa de un determinado patrón de desgaste luego de formular supuestos falsos. El más común de estos supuestos es que el peso de la sarta de perforación es transferido a la broca de manera eficiente. El WOB incide directamente en la ROP. Un ingeniero puede asumir que la selección deficiente de la broca está dificultando la ROP cuando en realidad el WOB, que es una función del diseño del BHA, es en efecto inferior al valor calculado. Por el contrario, cuando, el WOB es demasiado alto, la sarta de perforación y el BHA pueden flexionarse, lo que genera un pozo de mayor diámetro y produce vibraciones laterales destructivas ya que la broca angular se acciona y corta la pared del pozo. (Wiliamson y Lumbiski, 1986).

El plan direccional en Napo requiere mantener la tangente hasta la lutita de Napo Intermedia (lutitas laminadas negras (Salazar y Sáchez, 1989), e iniciar la caída de ángulo hasta llegar a TD al tope de Hollín. Para cumplir este objetivo, es necesario emplear un BHA tipo fulcro que permita contener la caída natural del ángulo generado en las lutitas de Napo, consideración que ya ha sido ampliamente estudiada por la compañía direccional y no forma parte de este estudio.

Los objetivos direccionales han sido alcanzados con éxito durante las operaciones de los pozos Coca K-40 y Coca K-41 llegando a cumplir el programa planteado. Se debe enfatizar que los lineamientos sobre la configuración de BHA que se detallarán, están orientados al correcto funcionamiento de la dinámica de fondo más que al cumplimiento del plan direccional. Estos lineamientos deben ser aplicados a pozos direccionales tipo "J" que tengan ángulos de inclinación similares a los pozos de estudio, es decir entre $30^{\circ}$ y $40^{\circ}$.

Las siguientes consideraciones fueron adoptadas en los pozos Coca K-40 y Coca K-41 en base del análisis de vibraciones realizado:

- Empleo de un motor con alto torque de salida. Esto con el fin de entregar mayor potencia en fondo para vencer el torque generado por la interacción broca-formación. En Ecuador el tipo de motor que se encuentra disponible tiene relación de lóbulos 7/8 con 4 etapas, lo cual cumple con este requerimiento.
- La configuración de tamaños de los estabilizadores debe ser de 12 inch en la camisa estabilizadora del motor y de 11.75 inch en el estabilizador que ha sido ubicado sobre el motor (García, 2008).

- Los ángulos de inclinación de los pozos están entre los $30^{\circ}$ y $40^{\circ}$ por lo que la configuración de estabilizadores se mantuvo en estos 2 pozos (Coca K-40 y Coca K-41), sin embargo, la dinámica de fondo puede cambiar si la disposición de estabilizadores se modifica en orden de alcanzar otro tipo de objetivos direccionales. La aplicación de los lineamientos se limita a pozos con perfiles direccionales en el rango ya establecido.

- Empleo de una broca de 5 aletas con cortadores de $19 \mathrm{~mm}$ y componentes secundarios controladores de torque, estos elementos son importantes, porque minimizan el riesgo de vibración torsional por penetración exagerada de los cortadores en la formación.

- Aplicación de tecnología reductora de fricción (tubería-paredes del hoyo), para mejorar así la transferencia de peso hacia la broca, obtener un torque más estable y con esto reducir los niveles de vibración torsional (Schlumberger). Se emplearon herramientas reductoras en los pozos Coca K-40 y Coca K-41.

- Para el caso específico de esta herramienta el caudal óptimo de trabajo oscila entre $600 \mathrm{gal} / \mathrm{min}$ y $900 \mathrm{gal} / \mathrm{min}$, con una caída de presión que varía entre 200 y 400 psi.

- El posicionamiento del reductor de fricción, debe ser por lo menos $1200 \mathrm{ft}$ sobre la broca para que la frecuencia de trabajo de la herramienta no afecte a la del MWD. Dicha frecuencia está entre 14.9-18.0 Hz, por tal razón, se debe configurar la frecuencia del MWD lo más baja posible.

- La ubicación exacta de la herramienta será determinada mediante simulación.

Con el caudal óptimo de trabajo, 800 psi y densidad del lodo de $11.5 \mathrm{lb} / \mathrm{gal}$, la caída de presión que genera la herramienta es 350 psi y frecuencia de trabajo de $16 \mathrm{~Hz}$.

La tabla 10, presenta un ejemplo de BHA que cumple con los requerimientos establecidos anteriormente. 
Tabla 10. Configuración de BHA Coca K-41.

\begin{tabular}{|c|c|c|c|c|c|}
\hline \multirow[t]{2}{*}{$\mathbf{N}^{\circ}$} & \multirow[t]{2}{*}{ Descripción } & $\begin{array}{l}\text { OD } \\
\text { (in) }\end{array}$ & $\begin{array}{c}\text { Max } \\
\text { OD } \\
\end{array}$ & \multirow{2}{*}{$\begin{array}{c}\text { Longitud } \\
-\quad(\mathrm{ft})\end{array}$} & \multirow{2}{*}{$\begin{array}{l}\text { Longitud } \\
\text { acumulada } \\
\text { (ft) }\end{array}$} \\
\hline & & ID (in) & (in) & & \\
\hline 1 & $\begin{array}{c}12 \text { 1/4” PDC Bit E1134 } \\
\text { - IADC M423 }\end{array}$ & $\begin{array}{c}8 \\
3.25\end{array}$ & 12.25 & 1.17 & 1.17 \\
\hline 3 & $\begin{array}{c}\text { Motor lóbulos } 7 / 8,4 \\
\text { etapas / Factor } 0.16, \mathrm{BH} \\
1.5^{\circ}, 12 \text { in Estabilizador }\end{array}$ & $\begin{array}{l}8.25 \\
6.25\end{array}$ & 12 & 29.72 & 31.89 \\
\hline 5 & Estabilizador & $\begin{array}{l}8.25 \\
2.84\end{array}$ & 11.75 & 7.25 & 43.14 \\
\hline 6 & LWD & $\begin{array}{c}8.38 \\
3\end{array}$ & 9.13 & 19.4 & 62.54 \\
\hline 7 & MWD & $\begin{array}{c}8.25 \\
5.9\end{array}$ & 8.38 & 27.35 & 89.89 \\
\hline 8 & Monel & $\begin{array}{l}8.25 \\
2.81\end{array}$ & 9.1 & 21.19 & 111.08 \\
\hline 10 & X-O (2 juntas) & $\begin{array}{c}9.5 \\
3.38\end{array}$ & 9.5 & 2.63 & 117.71 \\
\hline 11 & HWDP (33 juntas) & $\begin{array}{l}5 \\
3\end{array}$ & 6.5 & 1003.22 & 1120.93 \\
\hline 12 & Martillo Hidráulico & $\begin{array}{c}6.5 \\
2.75\end{array}$ & 6.5 & 31.55 & 1152.48 \\
\hline 13 & HWDP (3 juntas) & $\begin{array}{l}5 \\
3\end{array}$ & 6.5 & 92.41 & 1244.89 \\
\hline 14 & $\begin{array}{l}\text { Herramienta reductora } \\
\text { de fricción }\end{array}$ & $\begin{array}{l}6.75 \\
2.25\end{array}$ & 6.75 & 26.53 & 1271.45 \\
\hline 16 & HWDP & $\begin{array}{l}5 \\
3\end{array}$ & 6.5 & 30.61 & 1302.06 \\
\hline 17 & Intensificador & $\begin{array}{c}6.5 \\
2.75\end{array}$ & 6.5 & 32 & 1334.06 \\
\hline 18 & HWDP (3 juntas) & $\begin{array}{l}5 \\
3\end{array}$ & 6.5 & 91.32 & 1425.38 \\
\hline 19 & $\mathrm{X}-\mathrm{O}$ & $\begin{array}{l}6.5 \\
2.81\end{array}$ & 6.5 & 2.67 & 1428.05 \\
\hline
\end{tabular}

Se debe tomar en cuenta lo indicado por Cassaneli JP, Franco M. Pérez LC, Pinheiro C y Frenzel M en el momento de programar futuras operaciones de optimización, porque, el enfoque de tipo prueba y error respecto de los cambios del BHA permitían aliviar levemente las vibraciones axiales y laterales, pero exacerban la severidad del fenómeno de atascamiento deslizamiento. (Cassaneli, Franco, Pérez, Pinheiro y Frenzel, 2008).

\subsection{DEFINICIÓN DE PARÁMETROS}

En el paper Improving Drilling Performance by Applying Advanced Dynamics Models, se indica que los sensores de fondo de pozo y de superficie monitorean las vibraciones relacionadas con disfunciones. Sobre la base de las mediciones obtenidas con los sensores, los resultados de los modelos y la experiencia previa en operaciones de perforación en el campo. Los ingenieros ajustan los parámetros para optimizar la ROP y minimizar las vibraciones destructivas. (Dykstra, Neubert, Hanson y Meiners, 2001), se incorporará esta recomendación en los tres pozos estudiados.

En base al análisis obtenido del registro de vibraciones de los pozos (Coca K-29, Coca K-40, Coca K-41), en conjunto con la evaluación de la efectividad de las medidas preliminares adoptadas, se determinará el mapa de parámetros que mejor se adapte para perforar las subformaciones de Napo (sección 12 1/4 ") del Campo Coca.

\subsubsection{DETERMINACIÓN DE RPM Y WOB EN LA CALIZA "A" DE LA FORMACIÓN NAPO}

Mediante gráficas de contorno se mostrará la dependencia entre ROP, WOB y RPM en superficie para cada una de las sub-formaciones de Napo. Las zonas rojas representan la combinación de parámetros que generan mayor vibración.

Debido a la extensión del análisis y la gran cantidad de gráficas generadas, se detallará únicamente el proceso para obtener las RPM y WOB óptimos para perforar la caliza "A" (calizas micríticasmasivas y laminadas, con abundante cherts negros, presenta silicificación y abundante pirita (Jaillard, y otros, 2007)). Para realizar un análisis más exhaustivo sobre el comportamiento de las vibraciones, se dividió las sub-formaciones de Napo de acuerdo a la litología que contienen, como se 0 aprecia en la columna 2 de la tabla 3.

En las gráficas de contorno, se muestra la relación de las vibraciones en la broca y BHA con las RPM de superficie y WOB aplicados para perforar la lutita y caliza que conforman la caliza " $\mathrm{A}$ ".

Los valores de vibración torsional identificados son los típicos, sin embargo se trata de trabajar en zonas donde la vibración sea la menor posible. Se puede observar que con RPM entre $72-85$ y WOB entre 15 y $40 \mathrm{klb}$ se tiene la menor vibración torsional en la broca dentro de la caliza. Ver figura 8. 


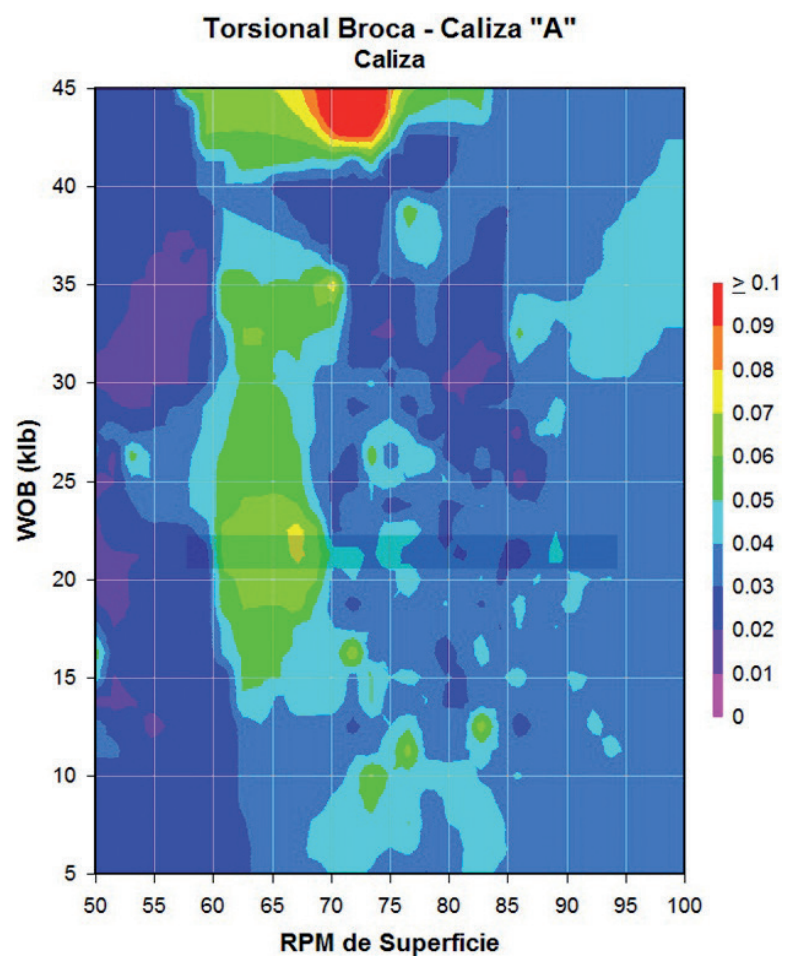

Figura 8. Vibración torsional broca caliza-caliza "A".

$\mathrm{Al}$ analizar la lutita que contiene la caliza " $\mathrm{A}$ ", se observa que con RPM de superficie entre 70-85 y WOB mayor a $25 \mathrm{klb}$ se generaron niveles más bajos de vibración torsional en la broca. Ver figura 9 .

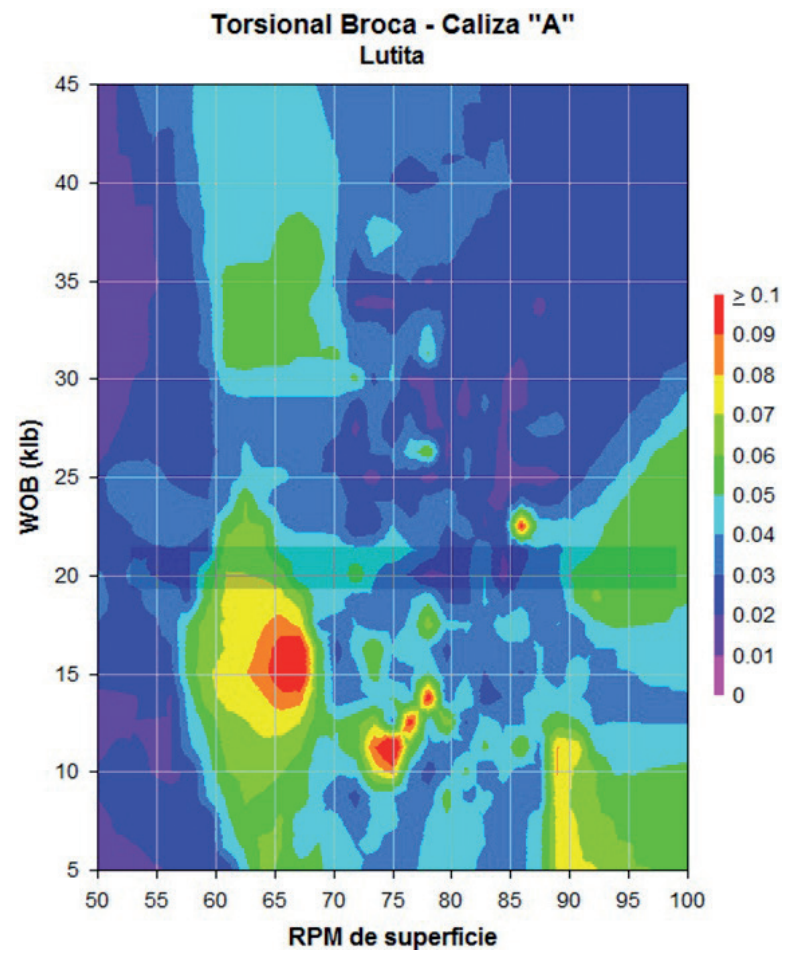

Figura 9. Vibración torsional broca lutita-caliza "A".
Para el BHA, a bajas RPM, la vibración torsional es alta a cualquier valor de WOB en la caliza y los niveles más bajos de vibraciones, se obtiene al superar las 70 RPM y WOB entre 24-35 klb. Ver figura 10.

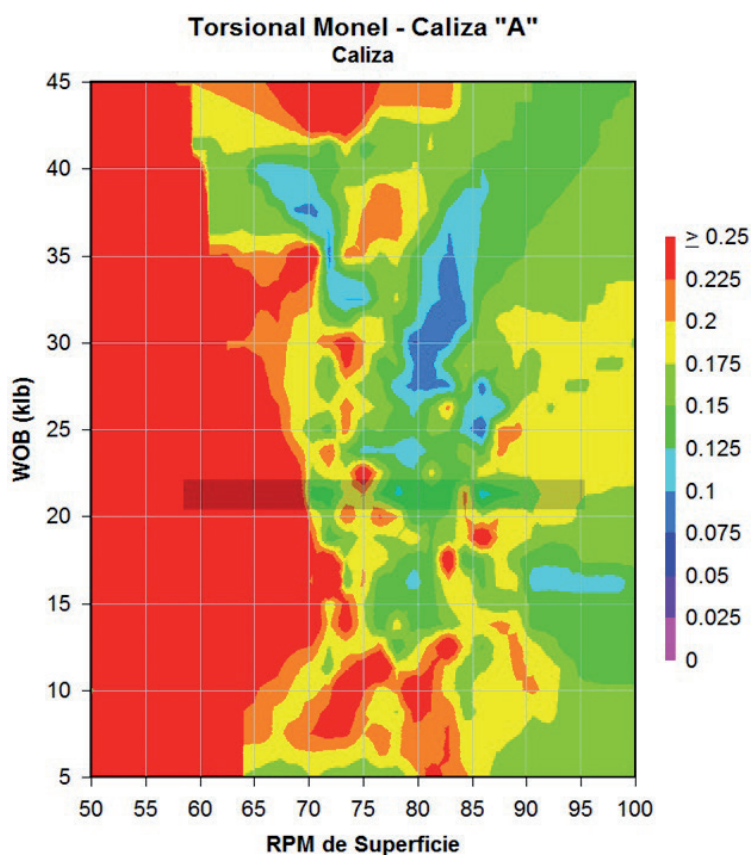

Figura 10. Vibración torsional monel caliza-caliza "A".

En los paquetes de lutita de la caliza "A", se mantiene la tendencia de altos niveles de vibración con bajas RPM a cualquier valor de WOB. Para trabajar con una vibración torsional leve, se debería aplicar más de 70 RPM y el WOB debería variar entre 25-35 klb. Ver figura 11.

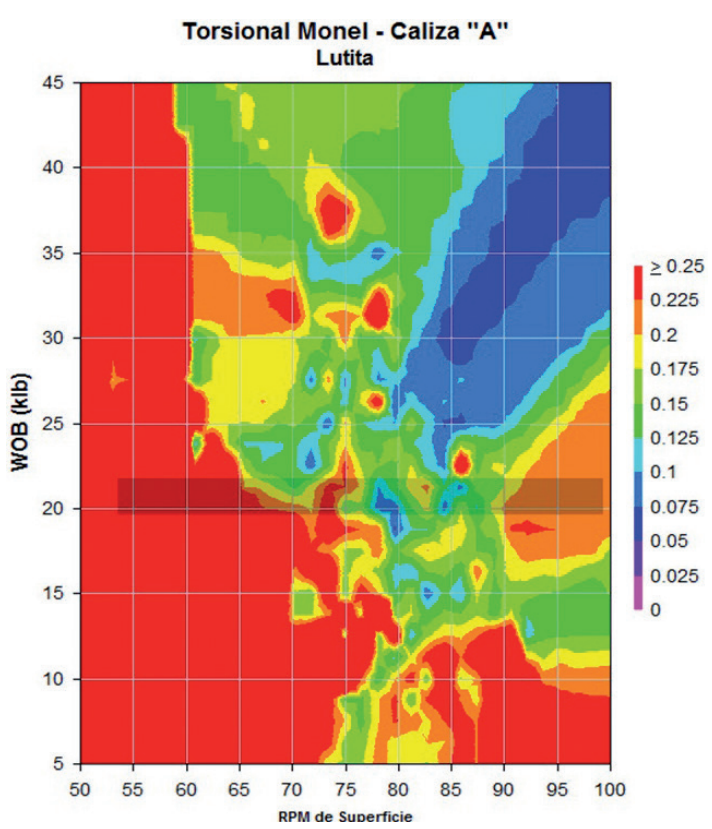

Figura 11. Vibración torsional monel lutita-Caliza "A". 
Además, al observar la relación del desempeño con los parámetros aplicados, considerando que las RPM oscilan entre 65 y 77, en conjunto con WOB mayor a 30 , se obtienen las más altas tasas de penetración en los paquetes de caliza. Ver figura 12 .

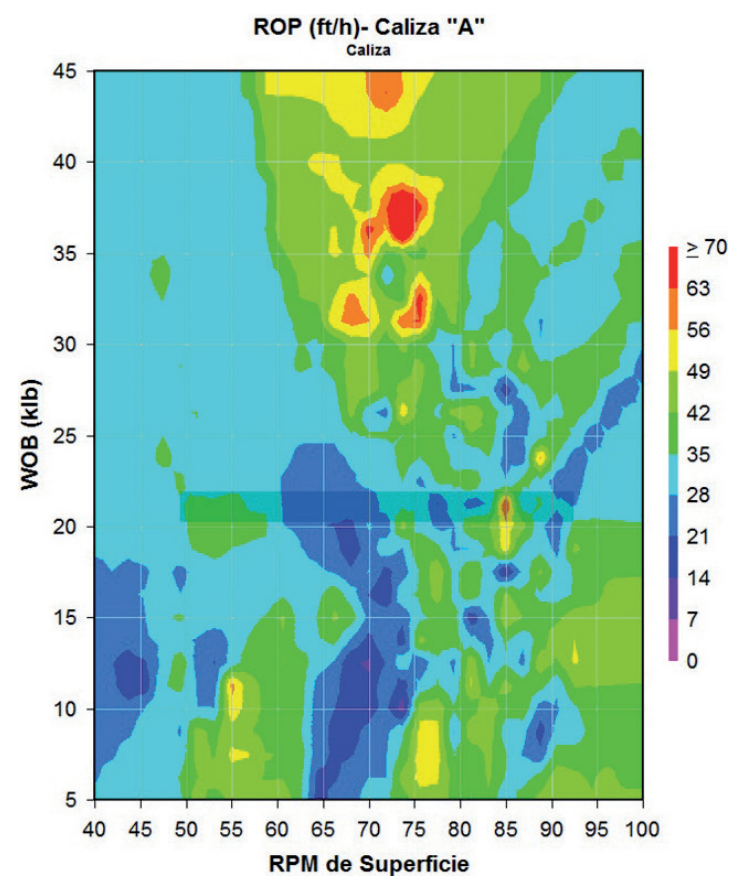

Figura 12. ROP caliza-caliza " $A$ ".

En los paquetes de lutita se obtiene las tasas de penetración más altas, con valores de RPM entre 60 y 77 y variaciones de WOB mayores a $32 \mathrm{klb}$. Ver figura 13.

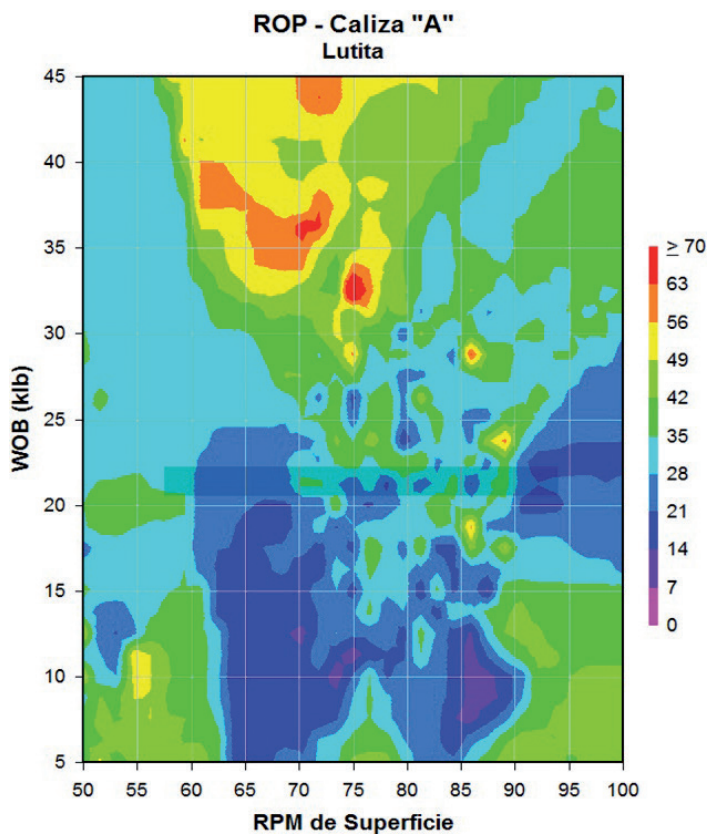

Figura 13. ROP caliza-caliza " $A$ ".

\subsubsection{PARÁMETROS PARA PERFORAR LA FORMACIÓN NAPO}

El procedimiento realizado para encontrar las RPM y el WOB óptimos para peforar la caliza "A" (descrita en la tabla 11) fue el utilizado para detallar los parámetros del resto de sub-formaciones de Napo.

Finalmente, con los parámetros establecidos para la broca y BHA según la litología de la subformación Napo, se realizó una intersección de los parámetros en común que generen menor vibración torsional y lateral de la mano con un alto desempeño.

La tabla 12, presenta el mapa de parámetros final, los cuales al implementarse podrían presentar pequeños intervalos en los que por diversas situaciones operacionales se puedan modificar.

Tabla 12. Mapa de parámetros para perforar Napo.

\begin{tabular}{|c|c|c|c|c|c|}
\hline \multirow{2}{*}{ Sub-Formación } & \multirow{2}{*}{ Litología } & \multicolumn{2}{|c|}{ WOB } & \multicolumn{2}{|c|}{ RPM } \\
\hline & & Min & Max & Min & Max \\
\hline \multirow{2}{*}{ Lutita Napo Superior } & Lutita & 25 & 37 & 75 & 80 \\
\hline & Caliza & 25 & 37 & 75 & 80 \\
\hline \multirow{2}{*}{ Caliza "M1" } & Lutita & 28 & 38 & 75 & 85 \\
\hline & Caliza & 25 & 35 & 80 & 85 \\
\hline \multirow{2}{*}{ Caliza "M2" } & Lutita & 27 & 40 & 75 & 85 \\
\hline & Caliza & 27 & 37 & 75 & 80 \\
\hline \multirow{2}{*}{ Caliza "A" } & Lutita & 30 & 35 & 70 & 75 \\
\hline & Caliza & 32 & 35 & 72 & 77 \\
\hline \multirow{2}{*}{ Arenisca "U" } & Lutita & 28 & 40 & 75 & 80 \\
\hline & Arenisca & 28 & 37 & 75 & 80 \\
\hline \multirow{2}{*}{ Lutita Napo Intermedia } & Lutita & 27 & 45 & 75 & 85 \\
\hline & Caliza & 24 & 40 & 75 & 85 \\
\hline \multirow{2}{*}{ Caliza "B" } & Lutita & 24 & 40 & 75 & 85 \\
\hline & Caliza & 27 & 40 & 75 & 80 \\
\hline \multirow{2}{*}{ Arenisca "T" } & Lutita & 28 & 38 & 75 & 80 \\
\hline & Arenisca & 17 & 38 & 75 & 80 \\
\hline \multirow{2}{*}{ Lutita Napo Inferior } & Lutita & 27 & 38 & 75 & 85 \\
\hline & Caliza & 27 & 38 & 75 & 85 \\
\hline \multirow{2}{*}{ Hollín } & Lutita & 24 & 40 & 75 & 85 \\
\hline & Arenisca & 24 & 40 & 75 & 85 \\
\hline
\end{tabular}




\subsubsection{CAUDAL PARA PERFORAR LA FORMACIÓN NAPO}

La caliza por su dureza tiende a generar vibración torsional de mayor intensidad como se ha demostrado en este estudio, esto se debe a que requiere de una gran cantidad de energía para vencer su resistencia. Esta energía se suministra desde superficie en forma de RPM o caudal para el caso de un motor de fondo, por lo tanto, si se considera el factor vibración, un alto caudal es de gran ventaja, puesto que entregaría más RPM en fondo y se dispondría de suficiente energía para vencer el torque reactivo de esta litología y así reducir la intensidad de la vibración torsional.

Son varias las ventajas de manejar un alto caudal, sin embargo, existen limitantes al valor máximo que se puede emplear.

- Los rangos de trabajo que manejan las herramientas direccionales para su correcto funcionamiento. Para el caso de un motor con una salida de torque como el utilizando en este análisis, relación de lóbulos $7 / 8,4$ etapas, bent housing $1.5^{\circ}$ y factor 0.16 , se maneja un rango entre $400 \mathrm{gpm}$ y $880 \mathrm{gpm}$.

- Las pérdidas de presión por fricción generadas al conducir el fluido de perforación hacia el fondo del pozo a través de la tubería. La presión de superficie máxima con la que trabaja el taladro de perforación generalmente es de 3800 psi.

Realizar los cálculos hidráulicos es fundamental porque permiten conocer la presión que se esperaría tener con el BHA, considerando las caídas de presión por herramientas direccionales, MWD, LWD, Motor, la caída de presión de la herramienta de oscilación axial y el diferencial aplicado con el motor a la hora de perforar.

Los cálculos de hidráulica para perforar Napo a la profundidad de salida de la corrida $10964 \mathrm{ft}$, con el tipo de BHA empleado en el pozo Coca K-41 con 4 se los realizó valores de caudal y los resultados se presentan en la tabla 13, que resume la hidráulica.

El valor máximo de caudal que cumple con los requerimientos direccionales, no excede la presión de las bombas del taladro y permitirá obtener las ventajas ya expuestas es $800 \mathrm{gal} / \mathrm{min}$. Estos cálculos consideraron un peso de lodo de $11.5 \mathrm{lb} /$ gal, viscosidad plástica 22 cp y un punto cedente $26 \mathrm{lb} / 100 \mathrm{ft}^{2}$ que generalemte se utilizan para perforar Napo. La broca fue configurada con 7 boquillas de 16/32 de pulgada, obteniéndose un TFA de $1.374 \mathrm{in}^{2}$.
Tabla 13. Cálculos de Hidráulica.

\begin{tabular}{cccccc}
\hline $\begin{array}{c}\text { Tasa de Flujo } \\
\text { (gal/min) } \\
\text { Presión de }\end{array}$ & 780 & 790 & $\mathbf{8 0 0}$ & 810 & 820 \\
$\begin{array}{c}\text { Superficie (psi) } \\
\text { Equipo Superficie }\end{array}$ & 3651 & 3712 & $\mathbf{3 7 7 4}$ & 3837 & 3900 \\
$\begin{array}{c}\text { (psi) } \\
\text { Flujo de Broca } \\
\text { (gal/min) }\end{array}$ & 757 & 766 & $\mathbf{7 7 6}$ & 786 & 795 \\
$\begin{array}{c}\text { Caída de Presión } \\
\text { de Broca (psi) }\end{array}$ & 321 & 329 & $\mathbf{3 3 8}$ & 346 & 355 \\
$\begin{array}{c}\text { HP/ HSI Broca } \\
\begin{array}{c}\text { Velocidad Chorro } \\
\text { (ft/s) }\end{array}\end{array}$ & $142 / 120$ & $147 / 1.25$ & $\mathbf{1 5 3 / 1 . 3}$ & $159 / 1.35$ & $165 / 1.4$ \\
\hline
\end{tabular}

\subsubsection{PROBLEMAS DE} CONTROL ASOCIADOS

Los problemas de control asociados a la dinámica presentada de la sarta de perforación son los siguientes:

- Mantenimeinto de la velocidad del mecanismo rotario de la superficie constante.

- Reducción de las oscilaciones de atascamientodeslizamiento.

- Mantenimiento de condiciones óptimas de operación. (Navarro y Suárez, 2004)

La propuesta descrita en el Mapa de Parámetros para Perforar Napo (tabla 12), permite direccionar las soluciones bajo un esquema de control de parámetros de perforación y diseño de BHA.

\section{CONCLUSIONES}

- Se demostró que es posible atravesar toda la formación Napo con una sola broca y en una sola corrida.

- Las intercalaciones de caliza y arenisca de la formación Napo, representan un mayor potencial de generación de vibraciones debido a la alta compresibilidad que poseen. Mayores niveles de vibración torsional $\mathrm{y}$ lateral son registrados cuando se emplea un sistema rotatorio dirigible RSS, puesto que, no entrega la suficiente cantidad de energía a la broca para vencer el torque reactivo de la litología de Napo.

- El incremento de las RPM de fondo entregado por un motor, reducen significativamente los niveles de vibración torsional en la broca y vibración lateral en el BHA al tener que aplicarse menor cantidad de RPM en superficie, así queda demostrado que utilizar motor de fondo es un método eficiente para resolver los problemas de vibración torsional originados en la interacción broca-formación. 
- La adaptación continua de parámetros de perforación RPM y WOB permitió obtener una dinámica de fondo más equilibrada en conjunto con la mayor ROP en el último pozo de estudio, Coca K-41:

- Se logró reducir en un 51\% los niveles de vibración torsional en la broca para el pozo Coca K-40 y del $96 \%$ en Coca K-41 en los cuales se empleó motor respecto a Coca K-29 donde se utilizó un RSS.

- Se incrementó la ROP que pasó de $23 \mathrm{ft} / \mathrm{h}$ en el pozo Coca K-29 a $45 \mathrm{ft} / \mathrm{h}$ en Coca K-40 y a $48 \mathrm{ft} / \mathrm{h}$ en Coca K-41, es decir, un incremento del 109\% de la ROP comparado con el pozo inicial.

\section{BIBLIOGRAFÍA}

1. Ayala, D., Torres, F., Valencia, R. y Loaiza, M. (2016). Impacto del Tiempo no Productivo en Operaciones de Perforación y Análisis de los datos Mediante la Prueba de Chicuadrado. Fuentes El Reventón Energético, 11.

2. Baldock. J. (1982). Geology of Ecuador. (pág. 20). Quito: Dirección General de Geología y Minas.

3. Bady. P., Rivadeneira. M. y Barragán. R. (2004). La Cuenca Oriente: Geología y Petróleo. Quito: Editores Científicos.

4. Betson, H. y Smith, H. (1956). Rock-Bit Desing, Selection and Evaluation. API 56-288, 35.

5. Brelt, J., Warren, T. yBehr, S. (1990). Bit Whirl-A new Theory of PDC Bit Failure. SPE 19571-PA, 275.

6. Cassanelli, J., Franco, M., Pérez, L., Pinheiro, C. y Frenfel, M. (2008). Dynamic Simulation Solving Vibration/Stick-Slip Issues Achieves Record ROP, Pagoreni Field. Perú. Lima.

7. Centale, P., Chala, V., Meehan, R., Páez, L., Partin, U., Segal, S., Wu, S., Garret, I., Teggart, B., Tetley, B. (2011). El diseño de las barrenas: Desde arriba hasta abajo. Sugar Land: Oilfield Review, 17

8. DATALOG. (2001). Datalog Manual de Operaciones en el Pozo (Tercera ed.).

9. Dykstra, M., Neubert, M., Hanson, J. y Meiners, M. (Marzo de 2001). Improving Drilling
Performance by Applying Advanced Dynamics Models. SPE/IADC 67697-MS, 18.

10. Garret. I., Teggart. B. y Tetley. (2010). FEA Modeling system Delivers High-Angle Well Bore through Hard Formations. E\&P 83, no. 9.

11. García, R. (2008). Estudio del Rendimiento de la broca HCM605Z para la sección de 12 1/4" diseñada para la perforación de pozos horizontales en el campo Tarapoha. Guayaquil: Escuela Politécnica del Litoral.

12. Jaillard, E., Benqston, P., Ordoñez, M., Vaca. W., Dhondt, A., Suárez, J., Toro, J. (2007). Sedimentary record of terminal Cretaceous accretions in Ecuador. Journal of South America Earth Sciences.

13. Korin, I., Morris, W., Gómez, M., Achem, H., Rodriguez, J. y Oroña, J. (2015). Desarrollo e Implementación de un Sensor de Vibraciones. San Antonio.

14. Navarro, E. y Suárez R. (2004). Vibraciones en una Sarta de Perforación: Problemas de Control. (pág. 6). Mexico: CONACYT 35989-A.

15. National Oilwell Varco. (2007). A Guide to Drilling Vibration. Houston.

16. Salazar, F. y Sánchez, H. (1989). Evaluación de las Arcillas en la Formación Napo, Areniscas «U» $y$ «T» en la Cuenca Oriente. (pág. 158). Quito: Universidad Central del Ecuador.

17. Sananikone, P., Kamoshima, O. y White, D. (1992). A Field Method for Controlling Drillstring Torsional Vibrations. Proceedings of the IADC/ SPE Drilling Conference (págs. 443-452). IADC/SPE 23891.

18. Schlumberger. (s.f.). Sensores y Optimización de la Perforación. (pág. 16).

19. Williamson, J. y Lumbiski, A. (1986). Predicting Bottom hole Assembly Performance. IADC/ SPE 14764-PA, 13.

20.Xianping, S., Páez, J., Partin, U. y Agrinhorti, M. (2010). Decoupling Stick-Slip and Whirl to Achieve Breakthrough in Drilling Performance. IADC/SPE 128767-MS, 13. 